\title{
Sixty-four-slice computed tomographic coronary angiography in pseudoaneurysm of the ascending aorta: $A$ useful modality to supplement the diagnosis
}

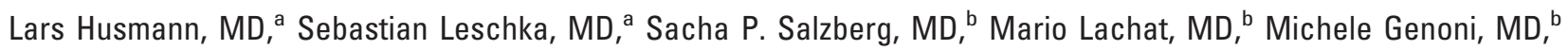 \\ Borut Marincek, MD, ${ }^{a}$ and Hatem Alkadhi, MD, ${ }^{a}$ Zurich, Switzerland
}

I nvasive coronary angiography (ICA) is the reference standard for preoperative workup of coronary arteries in patients undergoing cardiac surgery. In dedicated cases, however, ICA may fail when altered anatomy prevents selective catheterization of the coronary ostia. We present the imaging findings of a 58-year-old man with an anastomotic pseudoaneurysm of a composite graft in whom coronary opacification with ICA failed and computed tomography (CT) supplemented the diagnosis.

\section{Clinical Summary}

A 58-year-old man was admitted to our hospital for the workup of recurrent cerebral transient ischemic attacks. Thirteen years earlier, the patient had undergone composite graft implantation for the treatment of aortic regurgitation caused by a congenital bicuspid aortic valve and an aneurysmal dilatation of the aortic root. Transesophageal echocardiography revealed a covered perforation of the composite graft at the distal anastomosis. Because the recurrent ischemic attacks were attributed to emboli of thrombotic material within the pseudoaneurysm, the patient was scheduled for surgical revision of the composite graft. For preoperative workup of the coronary arteries, the patient underwent an ICA. Because of the particular anatomy of the aortic root, however, neither the right nor the left coronary artery could be selectively catheterized, and coronary artery opacification with nonselective injection of contrast material into the aortic root was considered insufficient (Figure 1). So that the coronary artery tree could be adequately visualized, a 64-slice CT (Sensation 64; Siemens AG, Forchheim, Germany) with retrospective electrocardiography gating after intravenous administration of $80 \mathrm{~mL}$ iodinated contrast material (Visipaque 320, Amersham Health, Buckinghamshire, United Kingdom) was performed. CT confirmed the covered rupture at the distal anastomosis of the composite graft. The rupture led to the creation of a pseudoaneurysm that surrounded the graft and eventually reached the level of the mechanical aortic valve prosthesis. Moreover, irregular thickening of the distal composite graft mem-

From the Institute of Diagnostic Radiology a and Clinic for Cardiovascular Surgery, ${ }^{\mathrm{b}}$ University Hospital Zurich, Switzerland.

Received for publication April 27, 2006; accepted for publication May 9, 2006.

Address for reprints: Hatem Alkadhi, MD, Institute of Diagnostic Radiology, University Hospital Zurich, Raemistrasse 100, 8091 Zurich, Switzerland (E-mail: hatem.alkadhi@usz.ch).

J Thorac Cardiovasc Surg 2006;132:e17-9

$0022-5223 / \$ 32.00$

Copyright () 2006 by The American Association for Thoracic Surgery

doi:10.1016/j.jtcvs.2006.05.002 brane adjacent to the rupture site indicated thrombotic material as the possible origin of the cerebral emboli. Sixty-four-slice CT demonstrated normal opacification of the coronary artery tree with no evidence of atherosclerotic wall changes and could reliably rule out significant coronary artery disease (CAD). It demonstrated, in addition, the relationship of the dissection-like membrane (ie, the composite graft) involving both coronary ostia (Figure 2). Subsequently, the patient underwent surgery that confirmed the covered rupture of the distal anastomosis creating a pseudoaneurysm and showed white thrombi along the membrane of the graft just adjacent to the rupture site. A tube graft was inserted at the site of the rupture, and both coronary arteries were reinserted. The patient had an uneventful postoperative course and was discharged to a rehabilitation facility on the 12 th postoperative day. In the 1-year clinical follow-up period after surgery, no additional cerebral event occurred.

\section{Discussion}

It is widely known that invasive angiography may be nondiagnostic and even hazardous in patients with aortic dissection when the catheter tip penetrates the false lumen. ${ }^{1}$ Altered anatomy such as in patients with congenital anomalies of the coronary arteries may be an additional reason for nondiagnostic catheter angiographic studies in that the anomalous vessel may be erroneously overlooked or assumed to be occluded if not selectively engaged. ${ }^{2}$ The patient in this report had a combination of these two conditions, harboring a dissection-like membrane, the composite graft, with a particular relationship of the coronary artery ostia, which led to the failure to selectively catheterize each coronary artery ostium.

Sixty-four-slice CT coronary angiography has been shown to have a high diagnostic accuracy for patients with CAD as compared with the reference standard ICA. ${ }^{3}$ Moreover, a recent study has demonstrated that multidetector-row $\mathrm{CT}$ is fast and accurate in the diagnosis of acute type A aortic dissection. ${ }^{4}$ Nevertheless, the clinical indications for 64-slice CT coronary angiography have not yet been established and remain a matter of debate. Most authors so far have suggested CT coronary angiography to be an alternative to ICA in patients with a low to intermediate pretest probability of CAD. ${ }^{3}$ This would also apply to patients with cardiac valvular disorders with no clinical evidence of CAD. In addition, CT has been shown to be a useful means in preoperative planning of minimally invasive cardiac procedures. ${ }^{5}$ The case presented herein may broaden the clinical applications of noninvasive 64slice CT coronary angiography to patients with pseudoaneurysms of the ascending aorta in whom complex anatomy prevents the preoperative workup of the coronary arteries with the invasive technique. CT additionally provides valuable anatomic information, especially in the setting of reoperative cardiac surgery. 


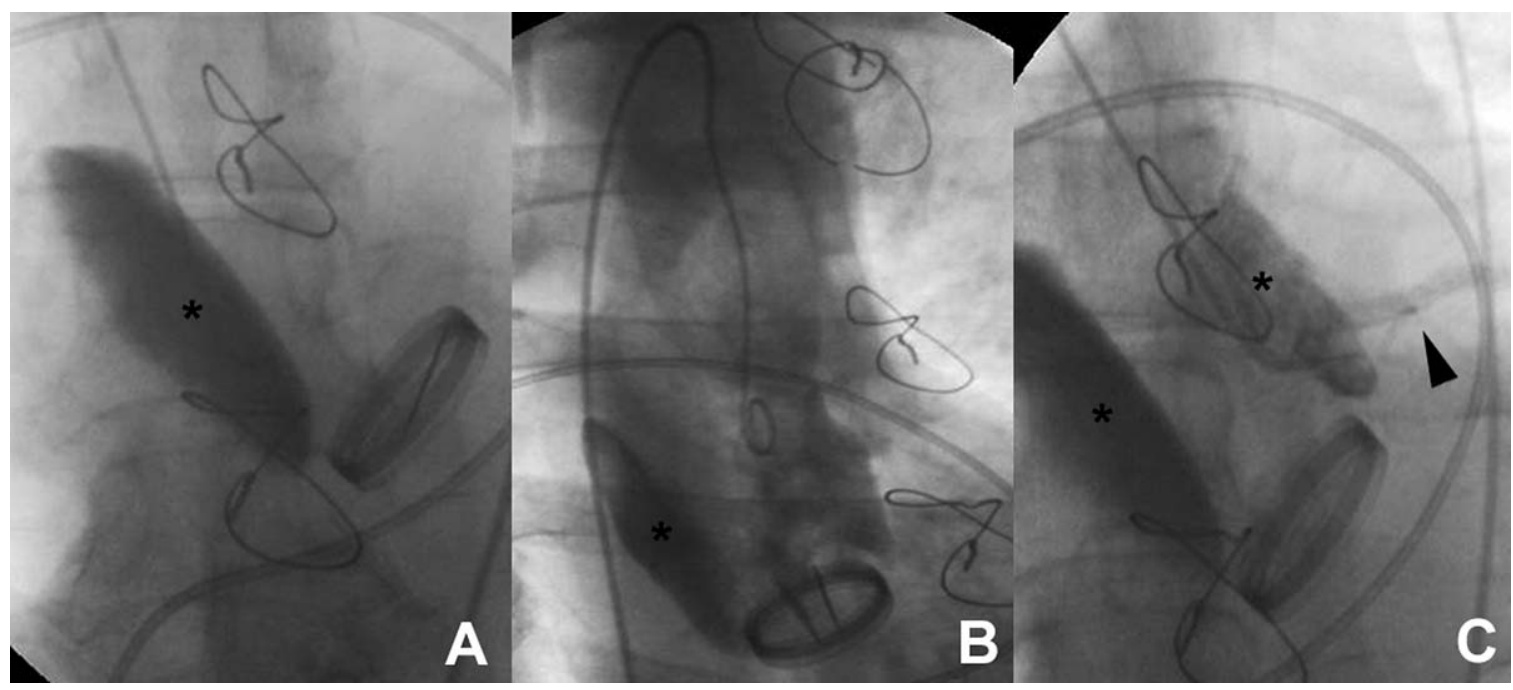

Figure 1. Invasive coronary angiography demonstrates the injection of contrast material into the pseudoaneurysm (asterisk) on the right side (A), into the composite graft (B), and into the pseudoaneurysm on the left side (C). Because of the exceptional anatomy of the aortic root, selective catheterization of both coronary ostia failed, and nonselective injection of contrast material yielded only insufficient opacification of the coronary arteries (black arrowhead: left main artery).

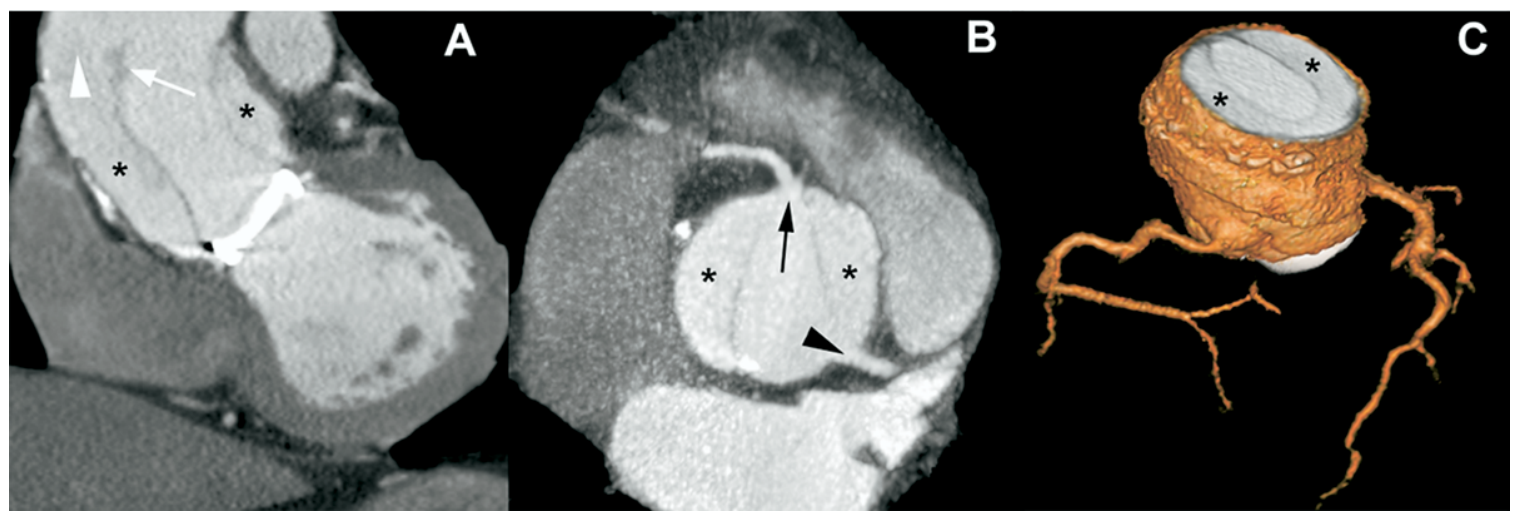

Figure 2. Sixty-four-slice CT after intravenous injection of contrast material with oblique coronal reconstruction (A), transverse reconstruction at the level of the coronary ostia (B), and volume rendered 3-dimensional image of the coronary artery tree (C). CT accurately depicts the covered rupture of the composite graft at the distal anastomosis creating a pseudoaneurysm (asterisk) that surrounded the graft and reached the level of the mechanical aortic valve prosthesis. Irregular thickening (white arrow) of the composite graft membrane adjacent to the rupture site (white arrowhead) indicated thrombotic material. The relationship of the origin of the left (black arrowhead) and right (black arrow) coronary arteries to the graft and pseudoaneurysm can be readily appreciated on the transverse image. The volume rendered 3-dimensional image demonstrates normal opacification of the coronary artery tree with no evidence of coronary stenoses. 


\section{References}

1. Abrams H. Dissecting aortic aneurysm: In: Abrams HL, editors. Abrams angiography. Volume 1, 3rd ed. Boston: Little, Brown; 1983, p. 441-66.

2. Shi H, Aschoff AJ, Brambs HJ, Hoffmann MH. Multislice CT imaging of anomalous coronary arteries. Eur Radiol. 2004;14:2172-81.

3. Leschka S, Alkadhi H, Plass A, Desbiolles L, Grunenfelder J, Marincek $\mathrm{B}$, et al. Accuracy of MSCT coronary angiography with 64-slice technology: first experience. Eur Heart J. 2005;26:1482-7.

4. Feuchtner GM, Schachner T, Friedrich G, Antretter H, Bonatti J, zur Nedden D. Acute aortic dissection with coronary ostium involvement and aortic valve regurgitation: three-dimensional visualization with multislice computed tomography. J Thorac Cardiovasc Surg. 2005;130: 587-8.

5. Ferdinand FD, Heiman M, Ben-Or S, Curtain AJ, Goldman SM. Three dimensional reconstruction of CT scan images to aid the cardiac surgeon. Semin Thorac Cardiovasc Surg. 2004;16:249-54. 